\title{
New onset vasovagal syncope following bariatric surgery: a case report
}

\section{Introduction}

This case discusses a young woman who presented with syncope and near syncope symptoms secondary to gastric bypass surgery. Her head tilt table test noted to be positive for VASIS type II a response. This is an interesting recorded case of such a presentation for vasovagal syncope.

\section{Case report}

47years old woman who recently went through gastric bypass surgery and was remarkably able to lose half of her body weight presented with monthly syncope episodes which are quite dramatic with abrupt loss of vision before complete loss of consciousness for anything from few seconds to a minute or so. These seem to be related to premenstrual and menstrual period although it is not always clear and according to the patient she also got them when she has been reclining, sitting, standing and walking. Otherwise, she is not aware of any other cardiac symptoms.

Clinically; she was in sinus rhythm with blood pressure of 134/86 $\mathrm{mmHg}$. An electrocardiography did not reveal any abnormalities. Patient was arranged for transthoracic echocardiogram and cardio memo, she also underwent the standardized protocol of upright tilt testing with trinitroglycerin (TNG) challenge which is; all were timed specifically with her menstrual period. Although the initial thought was that tilt table test is atypical for her history but the history of gastric bypass surgery raised the all possibilities. Her all lab investigations were within normal limits, the transthoracic echocardiogram as well as the cardio memo did not reveal any abnormalities but surprisingly, her tilt table test was positive for VASIS type II a response.

\section{Discussion}

It is not surprising then that bariatric surgery has become an increasingly popular treatment for obesity; commonly performed procedures include Roux-en-Y gastric bypass (RYGBP), biliopancreatic diversion with duodenal switch, and adjustable silastic gastric banding. ${ }^{1}$ Evidences from previous studies indicate that the reported incidence of neurologic-related complications has been increased, most notable those involving the somatic nervous system. ${ }^{2,3}$ Whereas, disruption in the autonomic nervous system and severe orthostatic intolerance have only rarely been reported following bariatric surgery. Orthostatic intolerance reflects an inability of the autonomic nervous system to adequately respond to the orthostatic stress of gravity which is characterized by insufficient cerebral perfusion resulting in symptoms upon standing and relieved by becoming spine. Symptoms may include syncope, near syncope and lightheadedness, It can be divided into three different groups: Reflex syncope, postural tachycardia syndrome, and autonomic failure. ${ }^{4}$ An observational retrospective study reviewed fifteen patients who had bariatric surgery for evaluation of orthostatic intolerance and the study demonstrated that approximately all patients $100 \%$ of them presented with lightheadedness, $11(73 \%)$ near-syncope and $9(60 \%)$ presented
Volume 8 Issue 2 - 2017

\author{
Matlooba AL Zadjali,' Faisal Alam, ${ }^{2}$ Matlooba \\ ALZadjali,' Faisal Alam,' \\ 'Ministry of Health, Directorate of Non-Communicable \\ Diseases, Oman \\ ${ }^{2}$ Sultan Qaboos University Medical College, Oman
}

Correspondence: Matlooba AL Zadjali, Ministry of Health, Directorate of Non-Communicable Diseases, Muscat, Sultanate of Oman, Oman, Tel 96895960998 , Emailmatlooba6@hotmail.com

Received: February 01, 2017 | Published: April 04, 2017

with syncope. All but one patient had a positive tilt table test with eight (53\%) having a neurocardiogenic response, three (20\%) having a dysautonomic response, and $(20 \%)$ having a postural tachycardia response. $^{5}$

While the exact mechanism or aetiology is not well described in the literature, but it could be due to weight loss-related reduction of baseline blood pressure and alteration of autonomic responsiveness. Interestingly, in our case report the patient had positive tilt table test for VASIS type II a response, this is suggesting that intermittent autonomic abnormalities resulting in hypotension and/or bradycardia resulted in orthostatic intolerance. ${ }^{6}$ Truly speaking, the exact incidence or prevalence of vasovagal syncope post Bariatric surgery is not known and from this case report it should be considered. Further investigations and underlying pathophysiology of vasovagal syncope following Bariatric surgery is warranted (Figure).

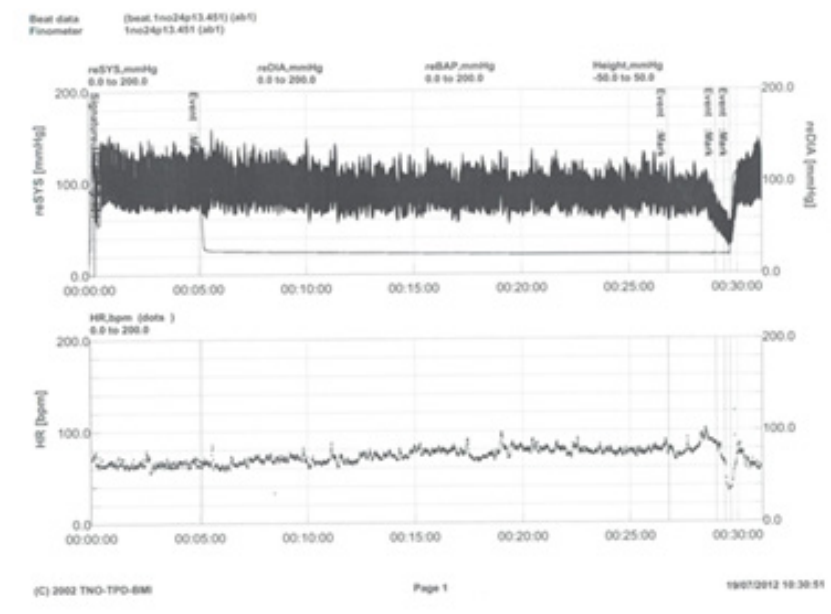




\section{Acknowledgments}

None.

\section{Conflicts of interest}

Author declares there is no conflicts opf interest.

\section{Funding}

None.

\section{References}

1. Santry HP, Gillen DL, Lauderdale DS. Trends in bariatric surgical procedures. JAMA. 2005;294(15):1909-1917.
2. Borgen JR. The neurologic complications of bariatric surgery. Arch Neurology. 2004; 61(8):1185-1189.

3. Abarbanel JM, Berginer VM, Osimani A, et al. Neurologic complication after gastric restriction surgery formorbid obesity. Neurology. 1987;37(2):196-200.

4. Grubb BP. Neurocardiogenic syncope and related disorders of orthostatic intolerance. Circulation. 2005;111(22):2997-3006.

5. Billakanty SR, Kligman MD, Kanjwal YM, et al. New-Onset Orthostatic Intolerance Following Bariatric Surgery. Pacing Clin Electrophysiol . 2008;31(7):884-888.

6. Hoeldtke RD, Dworkin GE, Gasper SR, et al. Sympathotonic orthostatic hypotension: A report of four cases. Neurology. 1989;39(1):34-40. 